\title{
Enhancement of Indoor Localization in WSN using PSO tuned EKF
}

\author{
Ravichander Janapati \\ Department of ECE, SR Engineering College, Warangal, India. \\ E-mail: ravi_chander_j@srecwarangal.ac.in \\ ch. Balaswamy \\ Department of ECE, QIS Engineering College Ongole, India. \\ E-mail: ch.balaswamy7@gmail.com \\ K.Soundararajan \\ Department of ECE, TKR Engineering College, India. \\ E-mail: soundararajan_jntucea@yahoo.com
}

\begin{abstract}
In Wireless Sensor Networks, nodes are positioned arbitrarily and finding location of nodes is difficult. In this network, the nodes need to know their location is important for indoor applications. In this applications signals are affected by various factors such as noise, multipath, NLOS etc. This impact on inaccurate location information of node, which leads finding path to the destination node is difficult. Cooperative location based routing is alternative solution for finding better path. In this paper a solution is proposed for effective route in indoor application of WSN. The proposed solution uses Particle Swarm Optimization assisted Adaptive Extended Kalman Filter (PSO-AKF) for finding location of nodes. In this mechanism, finding accurate position of node impact on network performance such as minimization of delay, location error and also minimizes complexity.
\end{abstract}

Index Terms - WSN, Kalman Filter, Extended Kalman Filter, Adaptive Extended Kalman Filter, Particle Swarm optimization, PSO assisted AKF, Localization.

\section{INTRODUCTION}

Wireless Sensor Networks (WSN) [1] is a collection of sensor devices, which performs operations such as sensing and computing in various applications. This network is used in wide range applications such as Industry [2], Agriculture [3], Civil infrastructure [4] and Security [5]. Recent developments in WSN are popularly used for indoor applications [6]. The deployment of sensor nodes in random positions, impact on finding accurate locations of nodes. Generally, GPS system is used in WSN for determining location of nodes. But, due to various reasons practical implementations involves lot of difficulties [7]. Finding location of nodes is essential for indoor applications.

The techniques such as Received Signal Strength (RSS) [8], Time of Arrival (TOA) [9], Time Difference of
Arrival (TDOA) [10] and Angle of Arrival (AOA) [11] are used currently for finding location. These solutions are ineffective due to noise and multipath effects in wireless communications. The localization algorithms can be classified in to two types' viz., centralized localization algorithm and distributed localization algorithm. In centralized localization algorithm the central node collects location information from all other nodes in network and perform localization task. The other techniques, all nodes in network execute algorithms and computes location information [12]. In this paper, the enhanced localization algorithm using Particle Swarm Optimization assisted Extended Kalman Filter (PSO-AKF) is proposed. After finding the locations, the communicate nodes are used locations and establish a route between source and destination node. The improvements are termed as Enhanced Localized Routing using PSO Assisted AKF.

Henceforth the paper is organized as follows section 2 explains the existing solutions from the literature. Particle Swarm Optimization (PSO), Kalman Filter (KF) are described in section 3. Section 4 illustrates the proposed mechanism in detail. The performance investigation and simulation outcomes are explained in section 5. Finally, section 6 concludes the paper.

\section{EXISTING SOLUTIONS}

The localization mechanism is important criteria in WSN for emerging applications. The existing solutions are finding locations based on GPS. But this method involves more complex and also consumes energy of a sensor node, which impact on poor network performance in terms of accuracy, Packet Delivery Ratio (PDR) and lifetime of network. In this section some of the existing solutions are explained.

The localization is important criteria in WSN for emerging applications. The existing solutions are finding locations based on GPS [13]. But this method is 
inaccurate and also consumes more energy of a sensor node, which leads network poor performance in terms of PDR, throughput and lifetime of network. In this section some of the existing solutions are explained.

GPS system can be used for localization of WSN in outdoor environment. It does not work well in the presence of obstacles and indoor environment [14]. Global Position system (GPS) gives inaccurate results and consumes more power [15]. Distributed Least Mean Square (DLMS) algorithm is used in the applications of localization and routing. DLMS algorithm is very simple in comparison with Distributed Recursive Least-Squares (DRLS) algorithm [16]. LMS algorithm has low SNR and less stable compare to DRLS algorithm and it offers good performance for very narrowband signals. DLMS algorithm requires external commands for control [17]. DRLS algorithm finds the location of nodes [18]. DRLS algorithm has faster convergence speed but this system is more complex [19].PSO is based on swarm intelligence. In PSO algorithm considers movement of group of birds, bees, school of fishes [20].The Kalman filter (KF) estimates unknown variables with precise values based on measurements over a time [21]. It has numerous applications in the field of signal processing,marine navigation, control of vehicles, aerospace. Extended Kalman Filter (EKF) is used to optimize the solutions of $\mathrm{KF}$ in nonlinear conditions.Extended Kalman Filter is optimized form of KF used in nonlinear environment [22].

The existing solutions in literature, finding the locations of node is inaccurate. In WSN, the location of node is important for finding the better routing path for communication. From the above information, in this paper Enhanced Localization algorithm is proposed and explained in section 4 .

\section{RELATED WORK}

The proposed method in this paper uses optimum mechanism i.e. Particle Swarm Optimization (PSO) which described in this section.

\section{A. Particle Swarm Optimization}

PSO is based on swarm intelligence. In PSO algorithm considers movement of group of birds, bees, school of fishes . Position of particle is denoted by $\mathrm{Xi}$ and velocity $\mathrm{Vi}$ then calculation of $\mathrm{Vik}+1$ using the following Eq.1 and 2 .

$$
\begin{gathered}
\mathrm{V}_{\mathrm{ik}+1}=\mathrm{W} \mathrm{\textrm {V } _ { \mathrm { ik } }}+\mathrm{c}_{1} \mathrm{r}_{1}\left(\mathrm{p}_{\text {best }}-\mathrm{X}_{\mathrm{ik}}\right)+\mathrm{c}_{2} \mathrm{r}_{2}\left(\mathrm{~g}_{\text {best }}-\mathrm{X}_{\mathrm{ik}}\right) \\
\mathrm{X}_{\mathrm{ik}+1}=\mathrm{X}_{\mathrm{ik}}+\mathrm{V}_{\mathrm{ik}}
\end{gathered}
$$

Vik $=$ velocity of node $i$ at iteration $k$ $\mathrm{Xik}=$ current position of node $\mathrm{i}$ at iteration $\mathrm{k}$

$c_{1}, c_{2}=$ acceleration constants

$r_{1}, r_{2}=$ randomly generated numbers consistently disseminated in the range $[0,1]$

$\mathrm{w}=$ inertia weight to control the scope of the search.

\section{B. Kalman Filter}

The Kalman filter (KF) estimates unknown variables with precise values based on measurements over a time . It has numerous applications in the field of signal processing,marine navigation, control of vehicles, aerospace. Extended Kalman Filter (EKF) is used to optimize the solutions of KF in nonlinear conditions.

\section{Extended Kalman Filter}

Extended Kalman Filter is optimized form of KF used in nonlinear environment which is given in Eq. 3 and 4 .

$$
\begin{aligned}
& x_{k+1}=f\left(x_{k}, k\right)+w_{k} \\
& z_{k}=h\left(x_{k}, k\right)+v_{k}
\end{aligned}
$$

Where $\quad \boldsymbol{x}_{\boldsymbol{k}}=$ state vector

$\boldsymbol{w}_{\boldsymbol{k}}=$ Process noise vector

$\widehat{\boldsymbol{Z}}_{K}=$ measurement vector

$\boldsymbol{V}_{\boldsymbol{K}}=$ measurement noise vector

\section{PROPOSED ENHANCED LOCALIZATION}

In this, the proposed Enhanced Localized Routing calculates nodes position using PSO Assisted AKF. Performance of the proposed method calculates the network performance by comparing with existing solution. Section 4.1 explains the proposed solution.

\section{A. Determination of location of nodes using PSO- Assisted AKF algorithm}

Consider a WSN in indoor environment where nodes are deployed randomly. In that some nodes do not know their position is known as agent nodes. Nodes which know their position is known as anchor nodes. Anchor nodes with known position $\left(\mathrm{x}_{1}, \mathrm{y}_{1}, \mathrm{x}_{2}, \mathrm{y}_{2}, \ldots \ldots \ldots \ldots\right)^{\mathrm{T}}$ find distance of unknown agent nodes using Received Signal Strength( RSS). Distance between anchor node and agent node is given by Eq.5

$$
\mathrm{P}_{\mathrm{i}}=\mathrm{d}_{\mathrm{i}}+\epsilon
$$

Where $d_{i}=\sqrt{\left(x-x_{i}\right)^{2}+\left(y-y_{i}\right)^{2}}$

$\epsilon=$ location error due to noise

$\left(\mathrm{x}_{\mathrm{i}}, \mathrm{y}_{\mathrm{i}}\right)=$ position of $\mathrm{i}_{\text {th }}$ agent node

$(\mathrm{x}, \mathrm{y})=$ position of anchor node

consider state space model of the location system Eq.6 is given by

$$
X_{K+1}=A X_{K}
$$

where $X_{K}=\left[X_{r K}, Y_{r K}\right]^{T} ; A=I_{2}=\left[\begin{array}{ll}1 & 0 \\ 0 & 1\end{array}\right]$ 
Measurement model of the system is given by Eq.7

$$
z_{k}=h_{k}\left(x_{k}\right)+v_{k}
$$

$z_{k}=$ Actual measurement of node $\mathrm{k}$.

$v_{k}=$ measurement noise

$$
h_{k}\left(x_{k}\right)=\sqrt{\left(x_{i k}-x_{k}\right)^{2}+\left(y_{i k}-y_{k}\right)^{2}}
$$

$H_{k}=$ Jacobian matrix of expected measurement is given in Eq.8

$$
H_{k}=\left[\frac{x_{i k}-x_{k}}{\sqrt{\left(x_{i k}-x_{k}\right)^{2}+\left(y_{i k}-y_{k}\right)^{2}}}, \quad \frac{y_{i k}-y_{k}}{\sqrt{\left(x_{i k}-x_{k}\right)^{2}+\left(y_{i k}-y_{k}\right)^{2}}}\right]
$$

\section{a. Adaptive Extended Kalman Filter}

In nonlinear environment EKF is used to estimate the location of nodes based on recursive equations. EKF consists of two phases time update, refinement phase viz.

\section{a) Time update phase}

Assume that initially no inputs are given to system.

$$
X_{K+1}=A X_{K}
$$

$X_{K}=$ state estimation of sensor at $\mathrm{k}$

$X_{K+1}=$ state estimation of sensor at $\mathrm{k}+1$

Predicted error covariance matrix is

$$
P_{K+1}=A P_{K} A^{T}
$$

$\boldsymbol{P}_{K+\mathbf{1}}=$ error covariance of $\boldsymbol{X}_{K+\mathbf{1}}$

$\boldsymbol{P}_{\boldsymbol{K}}=$ error covariance of $\boldsymbol{X}_{\boldsymbol{K}}$

\section{b) Measurement phase}

Innovation sequence gives the difference between predicted measurement and actual measurement. Innovation sequence is given in Eq.11.

$$
\widehat{y_{K}}=Z_{K}-h\left(x_{k}\right)
$$

Covariance of Innovation sequence is given in Eq.12.

$$
S_{K}=H_{k} P_{K} H_{K}^{T}+R_{K}
$$

$H_{k}=$ Jacobian matrix of expected measurement.

Updated state vector is given in Eq.13

$$
X_{K+1}=X_{K}+K_{K} \widehat{y_{K}}
$$

$k_{k}$ is known as kalman gain and calculated as follows in Eq.14

$$
k_{k}=H_{k}^{T} P_{K} s_{k}^{-1}
$$

Updated state covariance matrix is given in Eq.15

$$
P_{K+1}=\left(I-K_{K} H_{K}\right)
$$

From the Eq.13. it is observed that updated position state of nodes depends on innovation sequence. In indoor environment due to noise and multi path effects difference between estimated measurement and actual measurement is very high. Hence adapt noise covariance of innovation matrix to get accurate node positions.

$$
\begin{gathered}
R_{K} \rightarrow \alpha R_{K} \\
F I T=R O D=\frac{\operatorname{tr}\left(\hat{C}_{v k}\right)}{\operatorname{tr}\left(C_{v k}\right)}
\end{gathered}
$$

\section{b. PSO Assisted AKF}

PSO is an optimization mechanism which works on the behaviour of group of birds. In this proposed algorithm PSO is used to tune or assist covariance of innovation sequence.

1. Intialize population of particles with random position $\mathrm{X}_{\mathrm{i}}$ and random velocity $\mathrm{v}_{\mathrm{i}}$

2. Compute fitness of each particle using equation. Step by step procedure for PSO-AKF is given below

$$
F I T=R O D=\frac{\operatorname{tr}\left(\widehat{C}_{v k}\right)}{\operatorname{tr}\left(C_{v k}\right)}
$$

3. check the following conditions for evaluations

a) If $\mid$ FIT $\left(\mathrm{x}_{\mathrm{i}}\right)|<|$ FIT $\left(\mathrm{P}_{\text {best }}\right) \mid$ then $\mathrm{P}_{\text {best }}=\mathrm{x}_{\mathrm{i}}$

b) If $\mid$ FIT $\left(\mathrm{x}_{\mathrm{i}}\right)|<|$ FIT $\left(\mathrm{G}_{\text {best }}\right) \mid$ then $\mathrm{G}_{\text {best }}=\mathrm{x}_{\mathrm{i}}$

4. Velocity of each particle is given by

$$
\begin{gathered}
v_{i}=v_{i}+C_{1} \times \operatorname{rand}() \times \operatorname{rand}() \times\left(\text { Pbest }_{i}-x_{i}\right) \\
+C_{2} \times \operatorname{rand}() \times\left(\text { Gbest }-x_{i}\right)
\end{gathered}
$$

5. Update position of each particle using Eq.

$$
x_{i=} x_{i}+v_{i} \Delta t
$$

6. Repeat step 2 to 5 to get required FIT $\cong 1$

7. If FIT $\cong 1$ then output $=\mathrm{G}_{\text {best }}(\propto)$

8.Adapt noise covariance of innovation matrix to get accurate node positions using $\propto$.

$$
R_{K} \rightarrow \alpha R_{K}
$$

Pseudo code for the PSO-AKF is given in Fig.1 


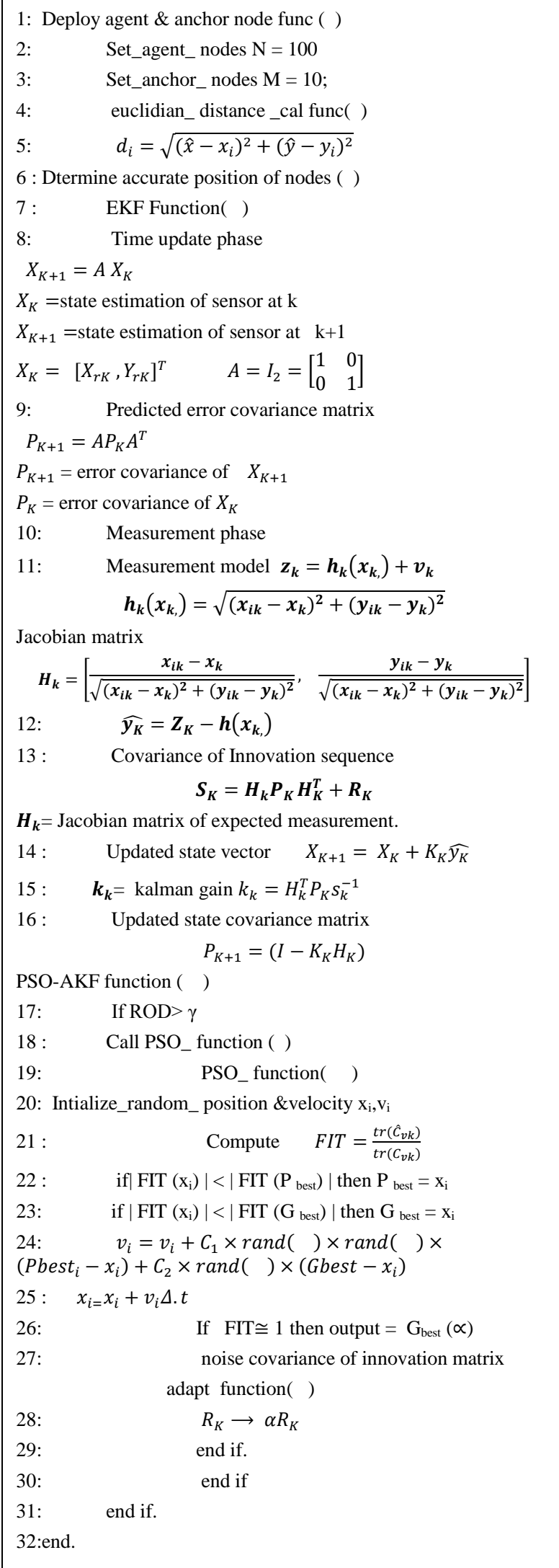

Fig.1. Pseudo code for PSO Assisted AKF

\section{Simulation Results AND ANALYSIS}

\section{A. Simulation setup}

For simulation, the sensor nodes are deployed in the area of $500 \mathrm{mX} 500 \mathrm{~m}$. In this, sensor nodes are classified as two types i.e. anchor nodes and agent nodes. Anchor nodes are known their positions primarily and agent nodes are not aware about their positions. The above deployment of sensor nodes is done using MATLAB. The transmission range of sensor node considers as $5 \mathrm{~m}$ and initial energy of nodes is 50 Joules. The simulation parameters are also listed in Table1.

Table 1. Simulation parameters

\begin{tabular}{|c|c|c|}
\hline Parameter & Symbol & Value \\
\hline Network size & $\mathrm{S}$ & 500mX500m. \\
\hline Environment & $\mathrm{E}$ & Indoor \\
\hline No.of anchor nodes & $\mathrm{Na}$ & 12 \\
\hline No.of agent nodes & $\mathrm{Na}$ & 120 \\
\hline Transmission range & $\mathrm{T}_{\mathrm{r}}$ & $5 \mathrm{~m}$ \\
\hline standard deviation & $\sigma$ & $\begin{array}{c}1 \% \text { of measured } \\
\text { distance }\end{array}$ \\
\hline Intial energy & $\mathrm{E}_{\mathrm{i}}$ & $5 \mathrm{~J}$ \\
\hline $\begin{array}{c}\text { Transmission power } \\
(\mathrm{W})\end{array}$ & $\mathrm{P}_{\mathrm{t}}$ & 2 \\
\hline $\begin{array}{c}\text { Distance of reference } \\
(\mathrm{m})\end{array}$ & $\mathrm{d}_{\mathrm{o}}$ & 2 \\
\hline Path loss exponent & $\alpha$ & 1.2 \\
\hline
\end{tabular}

The performance parameters used for the evolution is defined as follows.Performance of the proposed algorithm is evaluated by considering different scenarios and performance metrics. The performance metrics for analysis of results are defined below.

Cumulative Distribution Function (CDF): CDF is used to measure accuracy of localization system. If the accuracy of the two measuring systems are equal, then systems compared in terns of CDF. The system which reaches high probability values faster is considered as better system because its distance error is focused in small values.

Complexity: Average number of transmissions required to send the data from source to destination in specified intervals of time is known as complexity

Root Mean Square Error (RMSE): Difference between the estimated location value and and actual location value of localization algorithm. Equation 9 gives the formula to find RMSE value.

\section{B. Results analysis}

The proposed efficient localization routing tested with various parameters and results are analyzed. The proposed localization algorithm is analyzed and compared with EKF algorithm, kalman filter and least square algorithm.Fig.2 represents graph plotted for position versus CDF and results are compared among PSO-AKF algorithm, EKF algorithm, kalman filter and least square algorithm. In this least square algorithm performance is poor when compared with three 
algorithms. The reason is that noise and multipath effects shows impact on accuracy. The kalman filter performs better than least square method and less performance than PSO-AKF algorithm and EKF, due to KF does works well in nonlinear environment like noise, indoor environment. PSO-AKF algorithm performs better when compared with other algorithms, because of PSO assist the FIT parameter to find accurate positions.

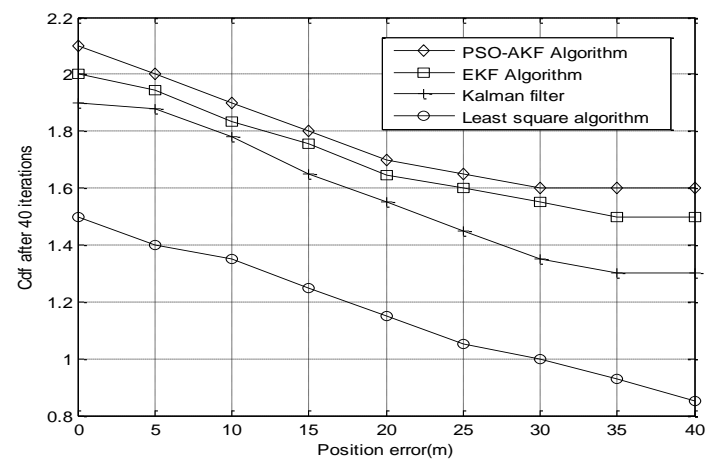

Fig.2. Performance comparison of different Algorithms by varying position error.

Fig. 3 represents graph plotted for iterations vs. avg. no. of transmissions per agent and results are compared among PSO-AKF algorithm without CRB, PSO-AKF with CRB. In this PSO-AKF algorithm without CRB performance is poor when compared with PSO-AKF with CRB. The reason is that CRB estimate lower bound of localization error and discards nodes which give inaccurate position information.

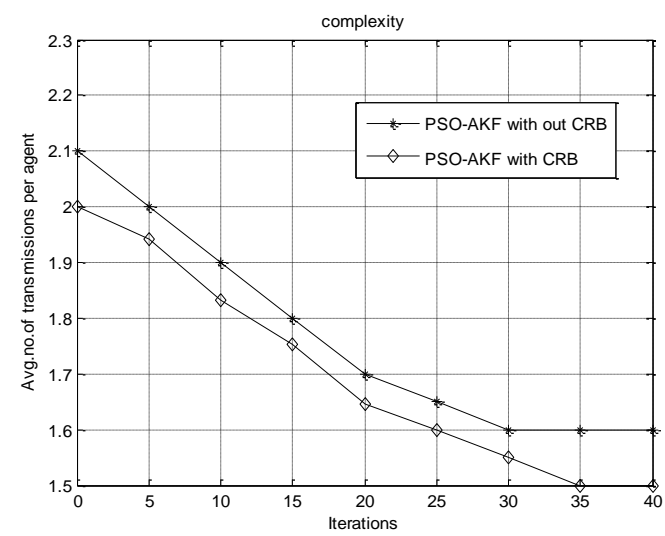

Fig.3. Performance comparison by varying number of iterations

Fig. 4 shows graph plotted for time vs. ROD and results are compared among PSO-AKF algorithm and EKF. PSO-AKF algorithm performance is better than EKF because PSO is used to assist AKF.

Fig. 5 represents graph plotted for No. of iterations Vs MSE of localization error and results are compared among PSO-AKF algorithm, EKF algorithm, KF and least square algorithm. In this least square algorithm performance is poor when compared with three algorithms. The reason is that noise and multipath effects shows impact on accuracy. The kalman filter performs better than least square method and less performance than
PSO-AKF algorithm and EKF, due to KF does works well in nonlinear environment like noise, indoor environment. PSO-AKF algorithm performs better when compared with other algorithms, because of PSO assist the FIT parameter to find accurate positions.

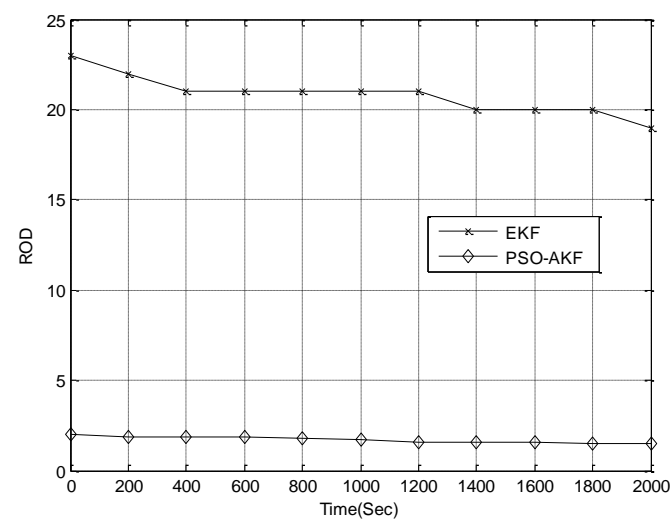

Fig.4. Performance comparisons by varying time

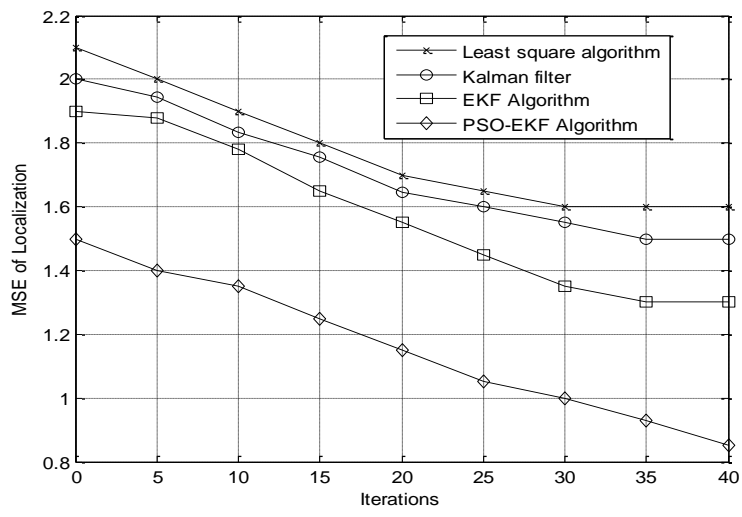

Fig.5. Performance comparison by varying number of iterations

In cooperative WSN all the agent nodes participated in the communication process. Fig. 6 shows number of anchor nodes Vs. CPU time. CPU time increases linearly as the number of anchor nodes increases. Hence select optimum references using CRB.

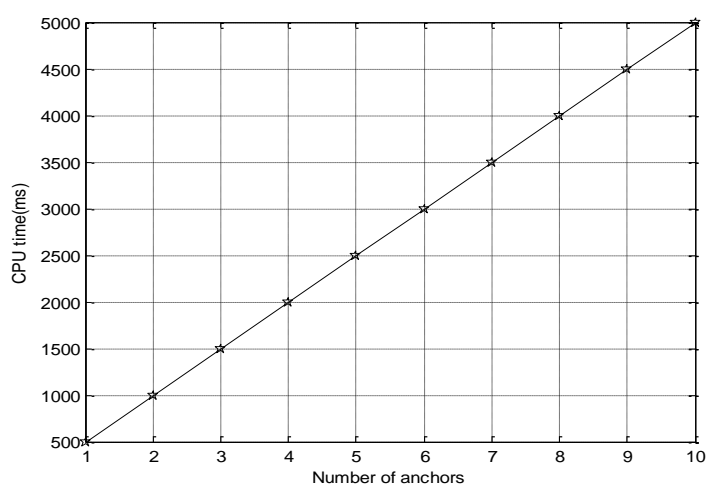

Fig.6. Performance comparison by varying number of anchor nodes

In a cooperative localization of WSN agent nodes also participated in localization process. Fig.7 shows the 
number of anchor nodes vs. location error. From the results it is observed that number of anchor nodes are less, location error is more. If number anchor nodes increase, location error decreases but complexity increases. At one particular point number of anchor nodes are more than 4 location error is same. Hence consider 4 anchors as optimum references.

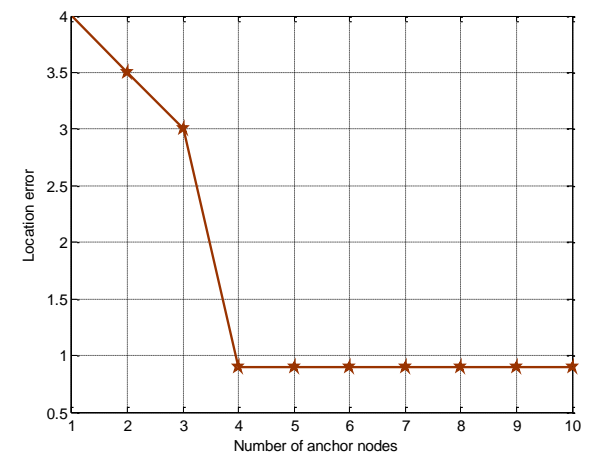

Fig.7. Performance comparison by varying number of anchor nodes.

The PSO tuned AKF algorithm performs better in comparison with other four algorithms. The reason is that proposed algorithm finds accurate nodes locations.

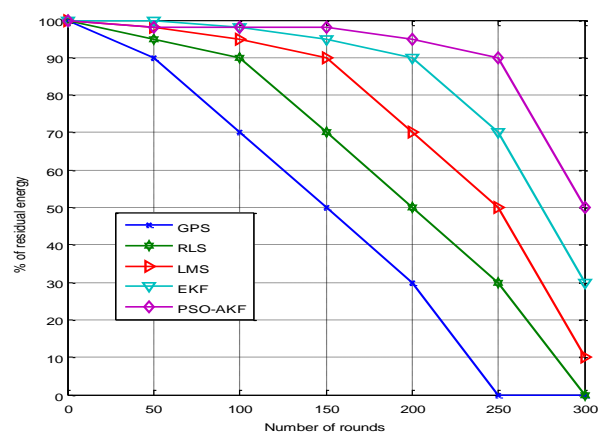

Fig.8. Number of rounds vs. residual energy

Fig. 8 shows a graph plotted for number of rounds vs. residual energy, results are compared among PSO-AKF, EKF, DRLS, DLMS and GPS in terms of residual energy. The performance of GPS is deprived in comparison with other four algorithms because of GPS is complex, consumes more power hence nodes died early. DRLS algorithm performs poor in comparison with DLMS, EKF and PSO-AKF because it is more complex and consumes more power. In this algorithm nodes died in 300 rounds. DLMS performs better in comparison with DRLS and GPS because it is less complex. Residual energy of nodes gradually decreased and $10 \%$ of energy remains at 300 rounds. EKF algorithm performs better in comparison with other algorithms because of less computational complexity. Residual energy of nodes gradually decreased and $30 \%$ of energy remains at 300 rounds. The PSO-AKF algorithm performs better in comparison with other four algorithms. The reason is that PSO-AKF, MSE is least and computational complexity is low. Hence residual energy of nodes improved.

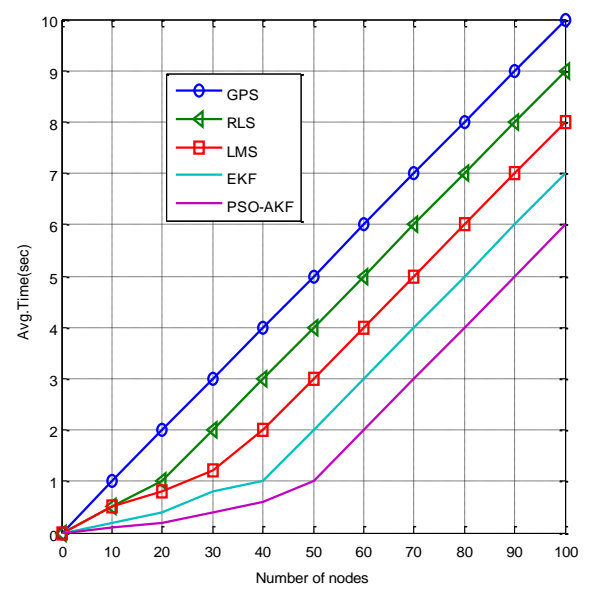

Fig.9. Number of nodes Vs. average time

Fig.9. shows graph plotted for number of nodes Vs. average delay and results are compared among PSO-AKF, EKF, DRLS, DLMS and GPS. Delay of network is increased with increasing number of nodes. The avg. delay of GPS is more when compared with other four algorithms because of GPS is more complex and requires more processing time. Avg. delay of DRLS algorithm is more in comparison with PSO-AKF, EKF and DLMS because of DRLS is complex in comparison with other algorithms. DLMS algorithm gives more avg. delay in comparison with EKF and PSO-AKF because of its SNR and stability is low. Avg. delay of DLMS is less in comparison with DRLS and GPS because it requires fewer computations. PSO algorithm gives less avg. delay in comparison with DRLS, DLMS and GPS because it is less complex, hence processing time is reduced. PSOAKF algorithm gives least avg. delay in comparison with EKF, DRLS, DLMS and GPS because its computational complexity and MSE is least.

\section{CONCLUSION}

In this paper, Enhanced localized routing in WSN using Particle Swarm Optimization assisted Adaptive Extended Kalman Filter (PSO-AKF) is proposed. To study the performance of proposed method, simulation study was conducted with various conditions. The proposed algorithm is compared with GPS, LMS, RLS, EKF and PSO-AKF. From the comparison result it was found that PSO-AKF performs better than other algorithms in terms of complexity, MSE and residual energy.

\section{ACKNOWLEDGEMENTS}

This work was supported by the [University Grant commission, India] under Grant [MRP-6233/15 (SERO/UGC].We gratefully thanks to Professors of ECE Department and Research \&Development Cell, JNTU,Anantapur for useful discussion \&suggestions for this research work. 


\section{REFERENCES}

[1] Akyildiz, Ian F., et al. "Wireless sensor networks: a survey." Computer networks 38.4 (2002): 393-422.

[2] A. H. Sayed, A. Tarighat, and N. Khajehnouri,BNetworkbased wireless location:Challenges faced in developing techniques for accurate wireless location information,IEEE Signal Process. Magzine.

[3] R. Fontana, E. Richley, and J. Barney,BCommercialization of an ultra-wideband precision asset location system, [ in Proc.IEEE Int. Conf. Ultra-Wideband (ICUWB),Nov. 2003, pp. 369-373.

[4] J. Caffery and G. Stuber, BRadio location inurban CDMA microcells, $[$ in Proc. IEEE Int.Symp. Personal, IndoorMobile Radio Commun.,Toronto, ON, Canada, Sep. 1995, vol. 2,pp. 858-862.

[5] Sathyan, T., \& Hedley, M. (2013). Fast and accurate cooperative tracking in wireless networks. Mobile Computing, IEEE Transactions on, 12(9), 1801-1813.

[6] Klonovs, J., Haque, M. A., Krueger, V., Nasrollahi, K., Andersen-Ranberg, K., Moeslund, T. B., \& Spaich, E. G. (2016). Monitoring Technology. In Distributed Computing and Monitoring Technologies for Older Patients (pp. 49-84). Springer International Publishing.Chicago

[7] Seco-Granados, G., López-Salcedo, J. A., Jiménez-Baños, D., \& López-Risueño, G. (2012). Challenges in indoor global navigation satellite systems: Unveiling its core features in signal processing. Signal Processing Magazine, IEEE, 29(2), 108-131.

[8] Tarrío, P., Cesana, M., \& Redondi, A. (2013). Energyaccuracy trade-offs for hybrid localization using RSS and inertial measurements in wireless sensor networks. Ad Hoc Networks, 11(6), 1874-1889.

[9] Shen, J., Molisch, A. F., \& Salmi, J. (2012). Accurate passive location estimation using TOA measurements. Wireless Communications, IEEE Transactions on, 11(6), 2182-2192.

[10] Wang, G., Li, Y., \& Ansari, N. (2013). A semidefinite relaxation method for source localization using TDOA and FDOA measurements. Vehicular Technology, IEEE Transactions on, 62(2), 853-862.

[11] Wang, Y., \& Ho, K. C. (2015). An Asymptotically Efficient Estimator in Closed-Form for 3-D AOA Localization Using a Sensor Network. Wireless Communications, IEEE Transactions on, 14(12), 65246535.

[12] Cota-Ruiz, J., Rosiles, J. G., Rivas-Perea, P., \& Sifuentes, E. (2013). A distributed localization algorithm for wireless sensor networks based on the solutions of spatially-constrained local problems. Sensors Journal, IEEE, 13(6), 2181-2191.

[13] Cadger, F., Curran, K., Santos, J., \& Moffett, S. (2013). A survey of geographical routing in wireless ad-hoc networks. Communications Surveys \& Tutorials, IEEE, 15(2), 621-653.

[14] N. Bulusu, J. Heidemann, and D. Estrin, "GPS-less Low Cost Out Door Localization for Very Small Devices,’Tech. rep. 00729, Comp. Sci. Dept., USC, Apr. 2000.

[15] Cheng, Bo, et al. "An accurate GPS-based localization in wireless sensor networks: a GM-WLS method." Parallel Processing Workshops (ICPPW), 2011 40th International Conference on. IEEE, 2011.

[16] Mateos, Gonzalo, Ioannis D. Schizas, and Georgios B. Giannakis. "Performance analysis of the consensus-based distributed LMS algorithm." EURASIP Journal on
Advances in Signal Processing 2009.1 (2009): 1-19.

[17] Cattivelli, Federico S., and Ali H. Sayed. "Diffusion LMS strategies for distributed estimation." Signal Processing, IEEE Transactions on 58.3 (2010): 1035-1048.

[18] Mateos, Gonzalo, Ioannis D. Schizas, and Georgios B. Giannakis. "Distributed recursive least-squares for consensus-based in-network adaptive estimation." Signal Processing, IEEE Transactions on 57.11 (2009): 45834588.

[19] Bertrand, Alexander, Marc Moonen, and Ali H. Sayed. "Diffusion bias-compensated RLS estimation over adaptive networks." Signal Processing, IEEE Transactions on 59.11 (2011): 5212-5224.

[20] Raghavendran, CH V., G. Naga Satish, and P. Suresh Varma. "Intelligent Routing Techniques for Mobile Ad hoc Networks using Swarm Intelligence."International Journal of Intelligent Systems and Applications 5.1 (2012): 81.

[21] G. Mallikarjuna Rao, Siva Prasad Nandyala, Ch.Satyanarayana,"Fast Visual Object Tracking Using Modified kalman and Particle Filtering Algorithms in the Presence of Occlusions", International Journal of Image, Graphics and Signal Processing (IJIGSP), Vol.6, No.10, pp.43-54, 2014.DOI: 10.5815/ijigsp.2014.10.06.

[22] Khan, M. W., Salman, N., Ali, A., Khan, A. M., \& Kemp, A. H. (2015, December). A comparative study of target tracking with Kalman filter, extended Kalman filter and particle filter using received signal strength measurements. In Emerging Technologies (ICET), 2015 International Conference on (pp. 1-6). IEEE

\section{Authors' Profiles}

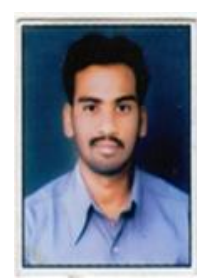

Ravichander Janapti is Research Scholar of Jawaharlal Nehru Technological University, Anantapur in the Area of Adhoc Wireless Sensor Networks and signal processing and Life Time Member of IETE. His Reaserch Papers published in Five International Journals and Six International Conferences.Now $\mathrm{He}$ is working as Senior Assistant Professor in SR Engineering college, Warangal,India.

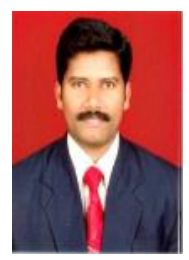

Ch. Balaswamy is Professor \&HOD Of ECE, QIS College of Engg\& Tech. His area of Interest is Mobile Adhoc Networks, Micro Processors \&Controllers and Embedded System.Hehas published eight International Journals and Six Researchpapers in National and International Conferences.

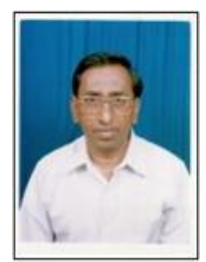

K. Soundararajan is professor \& director R\&D cell of TKR Engineering College. As a Researcher, he has successfully guided Nine $\mathrm{Ph} . D s$ in addition, to one in progress resulting in 27 International Jnl.s / Conf.Proc. , 32 national Jnls. / Conf. Proc. and 11 National seminars. 
How to cite this paper: Ravichander Janapati, ch. Balaswamy, K.Soundararajan,"Enhancement of Indoor Localization in WSN using PSO tuned EKF", International Journal of Intelligent Systems and Applications(IJISA), Vol.9, No.2, pp.10-17, 2017. DOI: $10.5815 /$ ijisa.2017.02.02 